\title{
Aspectos clínicos y psicopatológicos de la tartamudez precoz patológica
}

\author{
Dr. Carlos Almonte V.' ; Psicol. Sofía Lecara Z. ${ }^{2}$; Fonoaudjol. Edith Sehwalm A. ${ }^{3}$; \\ T. Ocupac. M. Astric Salen $\mathrm{Ch}^{4}$ \\ Clinical and psychopathological aspects of \\ early pathological stuttering
}

The authors analyse 13 children of both sexes who began stuttering before 7 ears of age and consulted during this period. A distinction is made between "physiological" stuttering and early "pathological" stuttering, focusing the study in the latter group. The different areas of neuropsychological development are analyzed, finding disturbances in speech and language organization in $77 \%$ of the children, $(n=10$ ) and difficulties in nother-child comunication in $100 \%$ of the cases. The authors pose that early "pathological" stuttering should be treated considering language and interpersonal relationships, since in a high percentage of patients jt becomes chronic, possibly persisting along school years, adolescence, and even adulthood, disturbing the development of personality and interpersonal relationships. This is not the case with "physiological" stuttering, which shows spontaneous remission.

(Key words: early pathological stuttering, development of language, family relations).

Durante los últimos doce años los autores han estudiado la tartamudez de 150 niños.

En el año 1986 se publicaron los resultados obtenidos en una muestra de 52 niños con tartamudez de curso crónico, en la cual se analizaron diversos aspectos clínicos y psicopatológicos ${ }^{1}$. Los hechos más significativos fueron que $71 \%$ de ellos había iniciado la tartamudez antes de los 7 aftos, $y$ ésta se asociaba con frecuencia a perturbaciones del desarrollo psicomotor, de las funciones perceptivomotoras y a trastornos específicos del aprendizaje de la lecto-escritura, así como a perturbaciones del desarrollo de la personalidad y de las relaciones interpersonales.

Estos hechos sugerian la conveniencia de enfocar un estudio en los menores de 7 años lo que

1. Psiquiatra lnfantil

2. Psicóloga.

3. Fonoaudióloga.

4. Teraperta Ocupacional.

Centro de Investigaciones del Desarrollo Integral del Niño. Servicio đe Neurología y Neurocinugía, Hospital Clínico de la Universidad de Chile y Servicio Otorrinolaringologia, Hospital Clínico de la Universidad de Chile. permitiría apreciar las características de la tartamudez en sus inicios, como tambièn el compro. miso de las funciones del desarrollo mencionadas

La información que se ofrece a continuación se refiere a las tartamudeces precoces patológicas, las que comienzan antes de los 7 años de edad, persisten más de 6 meses, se asocian a menudo a trastornos del habla y del lenguaje y tienden a la cronicidad, todo lo cual las distingue de las tartamudeces fisiológicas.

Los objetivos de este estudio fueron describir el estado evolutivo de las diferentes áreas del desarrollo neuropsíquico, su eventual compromiso en los ninos afectados de tartamudez precoz patologica y describir sus relaciones interperso. nales dentro del ámbito familiar.

\section{MATERIAL Y METODO}

Se diseñó un estudio exploratorio, clínico, descripti vo, en una muestra compuesta por todos los niños menores de 7 años de ambos sexos que iniçiaron tartamudeo antes de los 6 años y que consultaron en el Centro de Investigaciones del desarrollo integral del nifio (CIDIN), entre el 3 de marzo de 1982 y el 31 de mayo de 1983. La muestra estuvo constítuida por 11 varones y 2 mujeres, cuyas edades en el momento de consultar fluctuaban entre 4 y 6 años 9 meses; todos ellos habían 
iniciado la tartamude $z$ cntre los 2 y los 6 años. El tiempo de evolución del sintoma variaba entre 7 meses y 3 anos.

Se estudiaron diferentes aspectos del desarotio: en primer término se exaninaron las funciones neurologicas y maduración neuromotora; la psicomotricidad se evaluó mediante las pruebas de Oseretski revisión Guilmain modificada por Pierre Vayer correspondjente a la primera y segunda infancia ${ }^{3,4}$; para el estudio de las funciones perceptivo-motoras. se consideró el desarsolto de la organización perceptiva, la integración del esquema corporal y de las relaciones témporo-espaciales. Lat comunicación verbal se estudió con un cxamen fonoaudiológico, observando la respiración, los órganos articulatorios, la voz, habla, lenguaje y la conducta combnicativa. Esto $x$ llevó a cabo mediante el examen anátomo funcionaì de los órganos articulatorios, una evaluación linguística semicstructurada que contemplo la articulación, denominación, descripción $y$ narración $y$ la aplicación de la Prueba de rastreo de gramatica española, "Scteoning Test of Spanish Grammar" (STSG) de Toronto ${ }^{5}$. para analizar los aspectos gramaticales de la recepción y expresión del lenguaje.

Las funciones intelectuales fueron evaluadss con las prucbas de Wechsler (escala infantil) o con las pruebas de $B$ inet, de acucrdo a las características de cada caso Las funciones psicolinguísticas fueron estudiadas con prueba de Habilidad Psicolingústica de Illinois, de Kjrk y Mc Carthy.

Dentro del área de las relaciones interpersonales sólo se estudiaron las relaciones parento-fíliales. Pora evaluar estas relaciones se utilizaron datos de anammesis, obser vación de las interacciones en situación de entrcvista $y$ conclusiones de las pruebas proyectivas (CAT, dibujo de la tamilia).

\section{RESULTADOS}

En el estudio neuropsiquiátrico se repitió como antecente familiar más relevante el de la tartamudez en familiares adultos, que se елсолtró en $46,2 \%$ de los casos $(n=6)$. En cuanto a la ubicación en la fratria, no se registró ningún niño de ubicación intermedia en la familia: $61,5 \%$ $(n=8)$ eran los últimos hijos, 30,8\% $(n=4)$ hijos únicos y 7,7\% $(\mathrm{n}=1)$ primeros hijos. Entre los antecedentes personalés se registraron aquellos que pudieran tener alguna relación con la tarta. mudez $77 \% \quad(\mathrm{n}=10)$ habían iniciado el lenguaje en la edad comespondiente; $15,4 \%(\mathrm{n}=2)$ con discreto retardo y sólo $7,7 \%(\mathrm{n}=1)$ presentó franco retardo, iniciando el lenguaje después de los 36 meses.

Se registraron antecedentes de dislalias múltiples en $38,5 \%$ de los niños $(n=5)$. En la mayoría, $69,2 \%(n=9)$, la tartamudez se habia presentado en forma persistente $y$ sin intermitencias, en tanto que en $30,8 \%(n=4)$ se habian producido intermitencias. El sintoma era estacionario en $53,8 \%$ $(n=7)$, en $38,5 \%(n=5)$ tendia a acentuarse $y$ en $7,7 \%(n=1)$ habia ido atenuándose.

El tipo de tartamudez fue tónico-clónico en $84,6 \%$ ( $n=11)$; sólo en un caso era tónica y en otro clónica aisladas.

E1 grado de tartamudez, de acuerdo a la magnitud descrita en un trabajo anterior ${ }^{1}$, fue variable predominando las formas moderadas $y$ leves: $53,8 \%(n=5)$ y, $38,5 \%(n=5)$, respectivamente. Sólo se encontró un caso de tartamudez severa y ninguno muy severo.

Había conciencia de tartamudear o al menos de presentar dificuitades del habla en $69,2 \%$ $(\mathrm{n}=9)$ pacientes, los otros 4 no manifestaron conciencia de algún tipo de dificultad en la expresión oral.

Sólo $46,2 \%(n=6)$ habian recibido medicamentos, psicoterapia, o manejo ambienta\}, sin obtener resultados positivos. En $69,2 \%(n=9)$ de los casos el examen ncurológico fue normal, en los demás no se encontraron alteraciones leves que no configuraban sindromes neurológicos defünidos.

La neuromotricidad fue normal en $53,8 \%$ $(n=7)$ de los niños, en los otros 6 sc encontró inmadurez $(46,2 \%)$, que fue considerada leve en $30.8 \%(n=4)$ y moderada en un $15,4 \%(n=2)$ del total.

El estudio de la psicomotricidad dio por resultado normalidad del desarrollo psicomotor en el $61,5 \%(\mathrm{n}=8)$ de la totalidad del grupo examinado, en tanto que $38,5 \% \quad(n-5)$ presentaban diferentes formas de retardos parciales de la psicomotricidad.

Las funciones perceptivo-motoras fueron normales en $69,2 \%$ de los nitios $(\mathbf{n}=9)$, los restantes mostraron inmadurez leve o moderada.

El examen anátomo-funcional de los órganos articulatorios mostró la ausencia de alteraciones significativas orofaciales. La respiración era normal en $69,2 \%(n=9)$. Los 4 riños con alteraciones, correspondian a respiradores bucales y espasmos respiratorios durante la emisión del habla.

Los trastornos de la voz correspondían a alteraciones del timbre vocálico (disfonía); atteraciones de la resonancia vocal (nasalización); emisiones vocálicas espasmódicas y voz inspiratoria ocasional alcanzando a $61,5 \%(\mathrm{n}=8)$, los restantes ( $n=5)$ mostraban formación normal.

En el habla, además del transtorno del ritmo 
característico de la tartamudez se encontró en $77 \%(n=10)$ trastornos fonológicos, siendo el habla uno de los aspectos cuantitativamente más perturbados en todos los niños estudiados, Dentro de las alteraciones del habla, coexistían las dislatias propiamente tales con las deformaciones asistemáticas de la estructura de la palabra, producidas por asimilación, disimilación, inserción, contaminación, etc., semejantes a las observadas en los retrasos simples del lenguaje. Como cuadros aislados las "dislalias múltiples" representaron $30,4 \%(\mathrm{n}=4)$ de la muestra, el rotacismo $7,7 \%(n=1)$ y el ceceo $7,7 \%(n=1)$.

En el $77 \%$ de los casos $(n=10)$ habian alteraciones de la organización del lenguaje entre las que se consignaron trastornos morfosintácticos y semánticos, que se pusieron en evidencia en las praebas más complejas, que exigían un "corpus" verbal mâs amplio y semi-espontaneo. Los trastornos morfosintácticos aislados, akcanzaron a $53,8 \%(n=7)$. En el aspecto morfosintáctico expresivo lo más caracteristico fueron los agramatismos que ocurrieron en $38,5 \%(n=5)$ de los niños que se manifestaron como omisión de nexos gramaticales, alteraciones en el manejo de gramemas y de estructuras sintácticas. En lo receptivo se presentaron perturbaciones semejantes pero sólo en la decodificación de enunciados complejos.

Los trastornos semánticos ocurrieron en $69,2 \%(n=9)$ pacientes y se hicieron evidentes en lo expresivo y lo receptivo, caracterizándose por marcada pobreza léxico-semántica, contaminación de elementos dentro del mismo campo semántico, distorsiôn e incoherencia semántica en el relato. Estos desórdenes no fueron tan evidentes en el lenguaje espontaneo, pero si en los examenes especificos.

La conducta comunicativa fue adecuada a la situación de examen en $77 \%(n=10)$. Los otros 3 nifios, tenían trastornos caracterizados por verborrea, oposicionismo, distractibilidad y dificultad en el contacto afectivo.

En el estudio de las funciones intelectuales se encontró $53,8 \%(n=7)$ con niveles normales 0 superiores a lo normal y $46,2 \%(n=6)$ de subnormalidad, considerando ésta entre cuocientes intelectuales (CI) de 70 a 89 . El funcionamiento intelectual de la mayoría de los niños, $61,5 \%$ $(n=8)$, presentó armonía $u$ homogeneidad entre rendimientos verbales y perceptivo-motores, en tanto que los demás mostraban disarmonia significativa, correspondiendo en la totalidad a una baja verbal.

La edad psicolingüistica fue igual o mayor que la edad cronológica en $61,5 \%(n=8)$ y menor en los restantes casos. No se encontró un perfil típico de las diferentes funciones examinadas por Ia prueba.

En el estudio de las relaciones parento-filiales los resultados obtenidos difieren según la procedencia de los datos, existiendo en general conciencia sobre la información lograda por la obser. vación de las interacciones y los resultados de las pruebas proyectivas. Sin embargo, los datos de ta anamnesis entregados por el niño y por los padres no siempre fueron coincidentes.

Para calificar las relaciones corno satis. factorias o insatisfactorias se usaron los criterios señalados en una comunicación anterior ${ }^{k}$.

De acuerdo a los datos de anamnesis de los padres, la relación padre-hijo era satisfactoria en $69,2 \%$ de los casos, insatisfactoria en $15,4 \%$ $(n=2)$ y no se obtuvieron datos de los otros dos. La relación madre-hijo fue satisfactoria en $53,8 \%$ $(n=7)$ e insatisfactoria en los demás casos. Según los datos propoŕcionados por los ninos, la relación padre-hijo fue satisfactoria en $53,8 \%(n=7)$; insatisfactoria en $23,1 \%(n=3)$ no consignada en $23,1 \%(n=3)$. La relación madre-hijo fue satisfactoria en $46,2 \%(n=6)$, insatisfactoria en el 46,2\% $(n=6)$, sin datos en el 7,7\% (n=1). La coincidencia entre la información del hijo y la de sus propios padres, sólo se dio en $46,2 \%(n=6)$.

La observación de las interacciones espontá. neas padre-hijo fueron consignadas como insatisfactorias en $61,5 \%(n=8)$ de los casos, satisfactorias en $7,7 \%(\mathrm{n}=1)$, los demás casos no ocurrieron. En esta misma situación las interacciones madre-hijo fueron insatisfactorias en todos los casos.

De los resultados obtenidos por las pruebas proyectivas, la relación padre-hijo fue insatisfactoria en $53,8 \%(n=7)$, satisfactoria en $30,8 \%$ $(n=4)$ y no se obtuvieron datos en dos pacientes. La relación madre-hijo se reveló insatisfactoria en $92,3 \%(n=12)$, no obteniéndose ciatos en el otro nin̄o.

\section{COMENTARIO}

Al comparar los resultados de esta muestra con los obtenidos en un trabajo anterior ${ }^{1}$, cuya 
muestra abarcaba desde los 3 a 19 años, se observaban algunos hechos interesantes que permiten individualizar mejor este grupo.

En el trabajo anterior la mayoría de los niños tenía conciencia de tartamudear, predominaban las formas mixtas de intensidad moderada, segujdas por las formas severas o muy severas. En esta muestra, la tercera parte no tenía conciencia de tartamudear y menos aún de presentar otro tipo de dificultad en la expresión verbal, predominando las formas mixtas de intensidad moderada, seguidas en frecuencia por las formas leves. En ambos grupos se da la tendencia a la agravación o mantención del síntoma. Todo esto nos permite postular que las experiencias negativas $y$ las secuencias interaccionales ansiógenas van -acentuando la conciencia de dificultad que se expresa en la fijación del sintoma, en la agravación de la intensidad de éste, en la dificultad de las relaciones interpersonales, lo que finalmente puede perturbar el desarrollo de la personalidad.

Al analizar los resultados obtenidos en el examen anátomo-funcional de los órganos articulatorios, no se observaron alteraciones. Por otra parte, con respecto al desarrollo neurológico, de la neuromotricidad y psicomotricidad, como de las funciones perceptico-motoras, se aprecia una gran proporción de nomalidad, en tanto que un porcentaje menor presenta inmadurez leve. Por el contrario, los datos en cuanto a voz, habla y organización del lenguaje, muestran esta área altamente comprometida, to que corrobora los hallazgos de otros autores ${ }^{6,7}$. Así, en la mayoria de los niños se revelan alteraciones de la voz, alteraciones fonológicas. trastornos de tipo morfosintáctico, semántico o ambos, viéndose a menudo comprometido el desarrollo cognitivo, especialmente en cuanto al pensamiento verbal. Esto plantea la existencia de condiciones bioló. gicas favorabjes a la aparición y mantención del sintoma como a la perturbación de los apren. dizajes que requieren de buen desarrollo verbal, por ejemplo la lecto-escritura. La determinante biológica también se ve avalada por la proporción de varones respecto a niñas que en esta muestra fue de $5: 1$ y los antecedentes familiares de tartamudez, presentes en alto porcentaje; por otfa parte, aunque en los antecedentes personales del niño a menudo no hay constancia de retardo en la aparición del lenguaje, los trastornos en la organización de éste son muy frecuentes.
En esta muestra, desde el punto de vista del niño y su entorno llama la atención la ubicación de estos niños en la fratría, ya que al iniciarse la tartamudez $92 \%(n=12)$ era hijo único o último, lo que en general favorece la sobreprotección: la sobre involucración emocional coarta la autonomáa y debilita la eficiencia del niño en su enfrentamien to a las diversas situaciones de vida propias de su edad. Esto explicaría que a diferencia de los niños mayores en que se encontraban situaciones especificas perturbadoras, en esta muestra no se encontraron situaciones acentuadoras de sintomas.

Al estudiar las relaciones familiares se observa gran variabilidad en los resultados, que dependen del procedimiento utilizado, ya sea que se interrogue al niño o a los padres, que se observen las interacciones, o que se utilicen pruebas proyectivas. Esto plantea la dificultad de las personas involucradas para enjuiciar objetivamente sus relaciones, siendo los resultados más concordantes los de los abservadores externos.

En la muestra de niños mayores las disfunciones familiares eran moderadas o severas y los patrones de comunicación rígidos, con gran resistencia al cambio. Por el contrario, en esta muestra a pesar del alto número de relaciones insatisfactorias, las disfunciones familiares eran leves, con alta proporción de dificultades de la comunicación verbal y no verbal, especialmente en la relación madre-hijo. Sin embargo, los patrones de comunicación eran más flexibles en la interacción.

Las características que individualizan a este grupo de niños menores de 6 años, permiten afirmar que existen tartamudeces precoces que tienden a hacerce crónicas, por lo eual aquellos niños que presentan el síntoma más allá de 6 meses, que tienen perturbaciones en la organización del lenguaje, antecedentes familiares de tartamudez prolongada, $y$ familias que no.estimulan el desarrolto emocional, social y de la comunicación deberían ser considerados de riesgo. Este riesgo estaria en dos plenos, el de la fijación del síntoma y el de la posible asociación de trastomos de aprendizaje y alteraciones en el desarrollo de la personalidad.

En este sentido, parecería cenveniente separar las tartamudeces precoces, para el tratamiento, en "fisiológicas" y "patológicas".

Las tartamudeces precoces "fisiológicas" podrian ser, a menudo, expresión de un momento 
crítico en la vida de niños con cierta fragilidad en el área del lenguaje; sería una forma de reaccionar, como ocurre en otros síntomas, a una situación de tensión. Estas podrían remitir solas al desaparecer el factor externo. Sin embargo, en otros casos el síntoma no remite solo ya que el entomo del nifo mantiene la situación desfavorable o comienza a reaccionar frente al sintoma del niño.

Este tipo de tartamudeces precoces requeririan orientaciones generales a los padres frente al síntoma mismo e indicaciones para favorecer el desarrollo normal del niño en las áreas del lenguaje, emocional, de las interacciones y de la integración social.

En las tartamudeces precoces "patológicas", existe un trastomo prolongado de la comunica. ción $\mathrm{y}$ por lo tanto requeririan las mismas orientaciones anteriores; si hubieran índices de perturbaciones del lenguaje ya sea en el examen clínico, en su rendimiento escolar, sería necesario una evaluación de funciones cognitivas y eventualmente un tratamiento en esta área. Además se requeriría de técnicas terapéuticas que estimularan el crecimiento personal, la comunicación verbal y no verbal, at habla y lenguaje espontáneo en un contexto protegido y directivo.

\section{RESUMEN}

Se estudian 13 nirios de ambos sexos portadores de tartamudez iniciada antes de los 7 años y que consuitaron en este período etario. Diferen- cian en este periodo la tartamudez fisiológica de la precoz patológica, focalizando el estudio en el último grupo. Se analizan las diferentes áreas del desarrollo neuropsicológico, comprobando en el $77 \%(n=10)$ perturbaciones en el habla y en la organización del lenguaje y en $100 \%$ dificultades en la comunicación madre-hijo. Se plantea que la tartamudez precoz patológica a menudo debe ser tratada en cuanto a organización del lenguaje y relaciones interpersonales ya que en un alto porcentaje se hace crónica, pudiendo persistir durante el periodo escolar, adolescencia e incluso adultez, perturbando el desarrollo de la personalidad y de las relaciones interpersonales. Esto no ocurriria con la tartamudez fisiológica que es de resolución espontánea.

\section{REFERENCIAS}

1. Almonte C., Lecaros S., Sepúlyeda G. et al: Tartamudez infanto-juvenil. Enfoque Multidisciplinario. Acta Psiquiat Psicol Am Lat 1986; 32: 297-306.

2. Berthodin M.L.: Le Bégaiment Précoce. Approche orthophophonique. Neuropsych de l'enfance 1979; 27: 265-267.

3. Vayer P.: El diálogo corporal. Barcelona Editorial Científico-médica. 1977; 20*41.

4. Vayer $P_{.:}$El niño frente al mundo, Barcelona. Editorial Científico-médica. 1977; 201-240.

5. Toronto A.: Screening Test of Spanish Grammar. Evanston III. Northwestern. University Press 1973.

6. Wall $M .$, Myers $F_{.:}$A review of linguistic factors associated with early childhood stuttering. J. Commun Disord 1982; 15: 441-449.

7. Homzie M.J., Lindsay J.S.: Language and young stutterer: a new look at old theories and findings. Brain Lang $1984 ; 22: 232-252$. 